\title{
A tight colored Tverberg theorem for maps to manifolds (extended abstract)
}

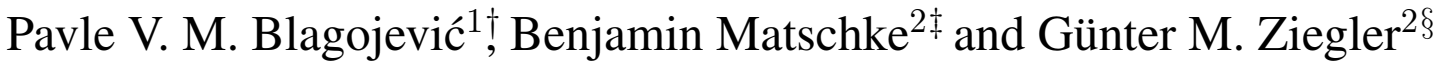 \\ ${ }^{1}$ Mathematički Institut SANU, Beograd, Serbia \\ ${ }^{2}$ Institut für Mathematik und Informatik, FU Berlin, Berlin, Germany
}

\begin{abstract}
Any continuous map of an $N$-dimensional simplex $\Delta_{N}$ with colored vertices to a $d$-dimensional manifold $M$ must map $r$ points from disjoint rainbow faces of $\Delta_{N}$ to the same point in $M$, assuming that $N \geq(r-1)(d+1)$, no $r$ vertices of $\Delta_{N}$ get the same color, and our proof needs that $r$ is a prime. A face of $\Delta_{N}$ is called a rainbow face if all vertices have different colors.

This result is an extension of our recent "new colored Tverberg theorem", the special case of $M=\mathbb{R}^{d}$. It is also a generalization of Volovikov's 1996 topological Tverberg theorem for maps to manifolds, which arises when all color classes have size 1 (i.e., without color constraints); for this special case Volovikov's proofs, as well as ours, work when $r$ is a prime power.

Résumé. Étant donné un simplex $\Delta_{N}$ de dimension $N$ ayant les sommets colorés, une face de $\Delta_{N}$ est dite arc-en-ciel, si tous les sommets de cette face ont des couleurs différentes. Toute fonction continue d'un simplex $\Delta_{N}$ de dimension $N$ aux sommets colorés vers une variété $d$-dimensionnelle $M$ doit envoyer $r$ points provenant de faces arc-en-ciel disjointes de $\Delta_{N}$ au mêmes points dans $M$; en supposant que $N \geq(r-1)(d+1)$, un ensemble de $r$ sommets de $\Delta_{N}$ doit être coloré à l'aide d'au moins deux couleurs. Notre démonstration requiert que $r$ soit un nombre premier.

Ce résultat est une extension de notre "nouveau théorème de Tverberg coloré", le cas particulier où $M=\mathbb{R}^{d}$. Il est également une généralisation du théorème de Tverberg topologique de Volovikov datant de 1996, pour les fonctions vers une variété, dont les classes de couleurs sont de taille 1 (c'est-à-dire sans contraintes de couleur). Dans ce cas particulier, la démonstration de Volovikov et la nôtre fonctionnent lorsque $r$ est une puissance d'un premier.
\end{abstract}

Keywords: equivariant algebraic topology, convex geometry, colored Tverberg problem, configuration space/test map scheme, group cohomology

\footnotetext{
${ }^{\dagger}$ The research leading to these results has received funding from the European Research Council under the European Union's Seventh Framework Programme (FP7/2007-2013) / ERC Grant agreement no. 247029-SDModels. Also supported by the grant ON 174008 of the Serbian Ministry of Science and Environment. pavleb@mi.sanu.ac.rs

$¥$ Supported by Deutsche Telekom Stiftung. matschke@math. fu-berlin. de

$\S$ The research leading to these results has received funding from the European Research Council under the European Union's Seventh Framework Programme (FP7/2007-2013) / ERC Grant agreement no. 247029-SDModels. ziegler@math.fu-berlin.de
}

1365-8050 @ 2011 Discrete Mathematics and Theoretical Computer Science (DMTCS), Nancy, France 


\section{Introduction}

More than 50 years ago, the Cambridge undergraduate Bryan Birch [5] showed that " $3 N$ points in a plane" can be split into $N$ triples that span triangles with a non-empty intersection. He also conjectured a sharp, higher-dimensional version of this, which was proved by Helge Tverberg [15] in 1964.

In a 1988 Computational Geometry paper [2], Bárány, Füredi \& Lovász noted that they needed a "colored version of Tverberg's theorem". Soon after this Bárány \& Larman [3] proved such a theorem for $r N$ colored points in a plane where the number of overlapping faces $r$ is 2 or 3 . Moreover, they conjectured a general version for any higher dimension $d$ and any number of overlaps $r \geq 2$, offering a proof by Lovász for the case $r=2$ and any dimension $d$. A 1992 paper [17] by Živaljević \& Vrećica obtained this in a slightly weaker version, though not with a tight bound on the number of points. The proof relied on equivariant topology and beautiful combinatorics of "chessboard complexes".

Recently we proposed a new "colored Tverberg theorem", which is tight, generalizes Tverberg's original theorem in the case of primes and gives the best known answers for the Bárány-Larman conjecture.

Theorem 1.1 (Tight colored Tverberg theorem [7]) For $d \geq 1$ and a prime $r \geq 2$, set $N:=(d+$ $1)(r-1)$, and let the $N+1$ vertices of an $N$-dimensional simplex $\Delta_{N}$ be colored such that all color classes are of size at most $r-1$.

Then for every continuous map $f: \Delta_{N} \rightarrow \mathbb{R}^{d}$ there are $r$ disjoint faces $F_{1}, \ldots, F_{r}$ of $\Delta_{N}$ such that the vertices of each face $F_{i}$ have all different colors and the images under $f$ have a point in common: $f\left(F_{1}\right) \cap \ldots \cap f\left(F_{r}\right) \neq \emptyset$.

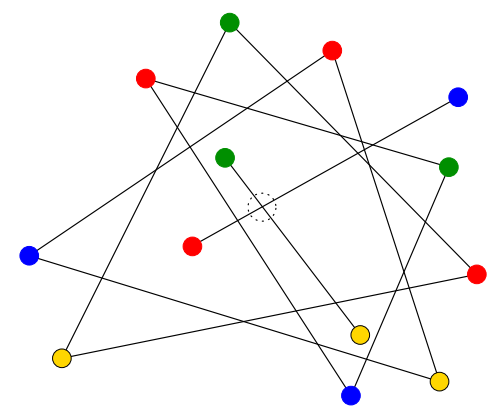

Fig. 1: Example of Theorem 1.1 for $d=2, r=5, N+1=13$.

Here a coloring of the vertices of the simplex $\Delta_{N}$ is a partition of the vertex set into color classes, $C_{1} \uplus \ldots \uplus C_{m}$. The condition $\left|C_{i}\right| \leq r-1$ implies that there are at least $d+2$ different color classes. In the following, a face whose all vertices have different colors, $\left|F_{j} \cap C_{i}\right| \leq i$ for all 1 , will be called a rainbow face. Figure 1 shows an example for Theorem 1.1

Theorem 1.1 is tight in the sense that it fails for maps of a simplex of smaller dimension, or if $r$ vertices have the same color. It implies an optimal result for the Bárány-Larman conjecture in the case where $r+1$ is a prime, and an asymptotically-optimal bound in general; see [7, Corollaries 2.4, 2.5]. The special case where all vertices of $\Delta_{N}$ have different colors, $\left|C_{i}\right|=1$, is the prime case of the topological Tverberg theorem, as proved by Bárány, Shlosman \& Szúcs [4]. 
In this talk we present an extension of Theorem 1.1 that treats continuous maps $R \rightarrow M$ from the a subcomplex $R$ of the $N$-simplex to an arbitrary $d$-dimensional manifold $M$ with boundary in place of $\mathbb{R}^{d}$. Here, $R$ is the rainbow subcomplex $\Delta_{N}$, which consists of all rainbow faces.

Theorem 1.2 (Tight colored Tverberg theorem for $M)$ For $d \geq 1$ and a prime $r \geq 2$, set $N:=(d+$ $1)(r-1)$, and let the $N+1$ vertices of an $N$-dimensional simplex $\Delta_{N}$ be colored such that all color classes are of size at most $r-1$. Let $R$ be the corresponding rainbow subcomplex.

Then for every continuous map $f: R \rightarrow M$ to a d-dimensional manifold, the rainbow subcomplex $R$ has $r$ disjoint rainbow faces whose images under $f$ have a point in common.

Theorem 1.2 without color constraints (that is, when all color classes are of size 1 , and thus all faces are rainbow faces and $R=\Delta_{N}$ ) was previously obtained by Volovikov [16], using different methods. His proof (as well as ours in the case without color constraints) works for prime powers $r$.

An extension of Theorem 1.2 to a prime power that is not a prime seems out of reach at this point, even in the case $M=\mathbb{R}^{d}$. Similarly, for the case when $r$ is not a prime power there currently does not seem to be a viable approach to the case without color constraints, even for $M=\mathbb{R}^{d}$. This is the remaining open case of the topological Tverberg conjecture [4].

Finally we remark that the restriction of the domain to a proper subcomplex of $\Delta_{N}$, as given by Theorem 1.2 appears to be a non-trivial strengthening, even though any partition can use only faces in $R \subset \Delta_{N}$ of dimension at most $N-r+1$. Let us give an example to illustrate that. Let $d=r=2$ and let $M$ be the 2-dimensional sphere. Then $N=3$ and we give the vertices of the tetrahedra $\Delta_{N}$ all different colors. Since the $N$-dimensional face of $\Delta_{N}$ is never part of a Tverberg partition, we might guess that the conclusion of Theorem 1.2 should hold true also for any map $f: \partial \Delta_{3} \rightarrow M$. However this is wrong: any homeomorphism $f$ gives a counter-example!

\section{Proof}

In this extended abstract we only consider the case when $f$ extends to a map $\Delta^{N} \rightarrow M$ on the whole simplex. If the given number of colors used to color the vertices is at least $d+3+\left\lfloor\frac{d}{r-1}\right\rfloor$ then the same proof will also work for non-extendable maps $f: R \rightarrow M$. Our proof of the general case of Theorem 1.2 needs some additional machinery due to Volovikov [16].

We prove Theorem 1.2 in this case in two steps:

- First, a geometric reduction lemma implies that it suffices to consider only manifolds $M$ that are of the form $M=\widetilde{M} \times I^{g}$, where $I=[0,1]$ and $\widetilde{M}$ is another manifold. More precisely we will need for the second step that

$$
(r-1) \operatorname{dim}(M)>r \cdot \operatorname{cohdim}(M),
$$

where cohdim $(M)$ is the cohomology dimension of $M$. This is done in Section 2.1 .

- In the second step, we can assume (1) and prove Theorem 1.2 for maps $\Delta_{N} \rightarrow \vec{M}$ via the configuration space/test map scheme and Fadell-Husseini index theory, see Sections 2.2 and 2.4 The basic idea is the following: Assuming that Theorem 1.2 has a counter-example, construct an equivariant map from it. Then we show using equivariant topology that such a map cannot exist.

In the second step we rely on the computation of the Fadell-Husseini index of joins of chessboard complexes that we obtained in [8, Corollary 2.6]. 


\subsection{A geometric reduction lemma}

In the proof of Theorem 1.2 we may assume that $M$ satisfy the above inequality (1) by using the following reduction lemma repeatedly.

Lemma 2.1 Theorem 1.2 for parameters $(d, r, M, f)$ can be derived from the case with parameters $\left(d^{\prime}, r^{\prime}, M^{\prime}, f^{\prime}\right)=\left(d+1, r, M \times I, f^{\prime}\right)$, where the continuous map $f^{\prime}$ is defined in the following.

Proof: Suppose we have to prove the theorem for the parameters $(d, r, M, f)$. Let $d^{\prime}=d+1, r^{\prime}=r$, and $M^{\prime}=M \times I$. Then $N^{\prime}:=\left(d^{\prime}+1\right)(r-1)=N+r-1$. Let $v_{0}, \ldots, v_{N}, v_{N+1}, \ldots, v_{N^{\prime}}$ denote the vertices of $\Delta_{N^{\prime}}$. We regard $\Delta_{N}$ as the front face of $\Delta_{N^{\prime}}$ with vertices $v_{0}, \ldots, v_{N}$. We give the new vertices $v_{N+1}, \ldots, v_{N^{\prime}}$ a new color. Define a new map $f^{\prime}: \Delta_{N^{\prime}} \rightarrow M^{\prime}$ by

$$
\lambda_{0} v_{0}+\ldots+\lambda_{N^{\prime}} v_{N^{\prime}} \longmapsto\left(f\left(\lambda_{0} v_{0}+\ldots+\lambda_{N-1} v_{N-1}+\left(\lambda_{N}+\ldots+\lambda_{N^{\prime}}\right) v_{n}\right), \lambda_{N+1}+\ldots+\lambda_{N^{\prime}}\right) .
$$

Suppose we can show Theorem 1.2 for the parameters $\left(d^{\prime}, r^{\prime}, M^{\prime}, f^{\prime}\right)$. That is, we found a Tverberg partition $F_{1}^{\prime}, \ldots, F_{r}^{\prime}$ for these parameters. Put $F_{i}:=F_{i}^{\prime} \cap \Delta_{N}$. Since $f^{\prime}$ maps the front face $\Delta_{N}$ to $M \times\{0\}$ and since $\Delta_{N^{\prime}}$ has only $r-1<r$ vertices more than $\Delta_{N}$, already the $F_{i}$ will intersect in $M \times\{0\}$. Hence the $r$ faces $F_{1}, \ldots, F_{r}$ form a solution for the original parameters $(d, r, M, f)$. This reduction is sketched in Figure 2
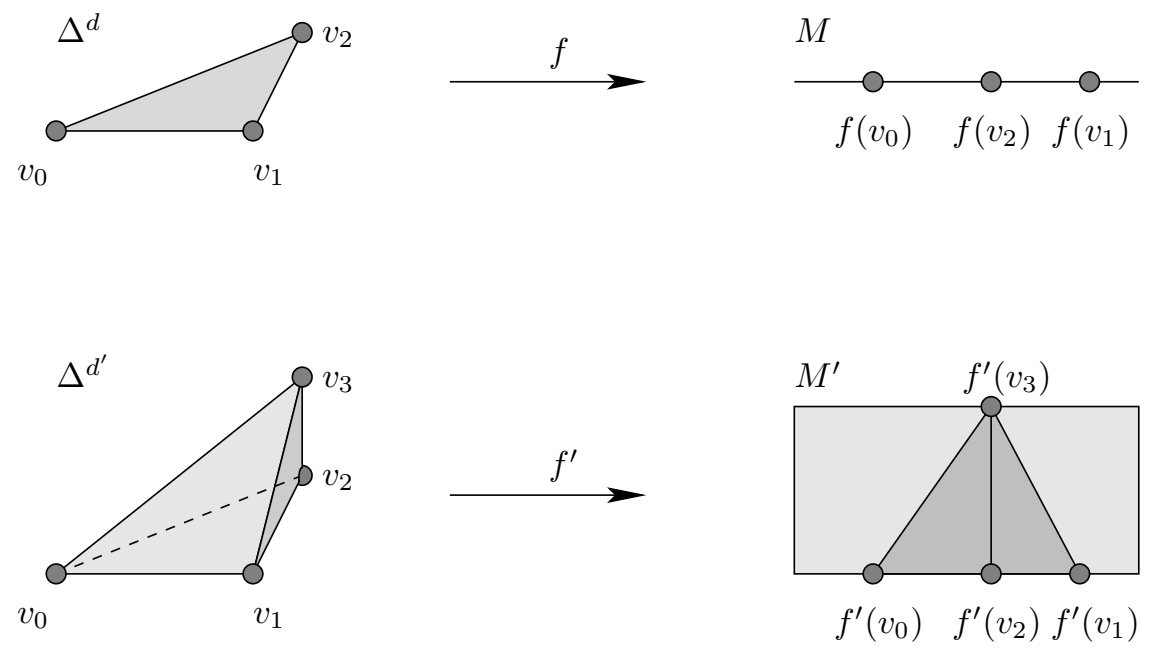

Fig. 2: Exemplary reduction in the case $d=1, r=2, N=2$.

If the reduction lemma is applied $g=1+\left\lfloor\frac{d}{r-1}\right\rfloor$ times, the problem is reduced from the arbitrary parameters $(d, r, M, f)$ to parameters $\left(d^{\prime \prime}, r^{\prime \prime}, M^{\prime \prime}, f^{\prime \prime}\right)$ where $M^{\prime \prime}=M \times I^{g}$. Thus $M^{\prime \prime}$ has vanishing cohomology in its $g$ top dimensions. Therefore $(r-1) \operatorname{dim}\left(M^{\prime \prime}\right)>r \cdot \operatorname{cohdim}\left(M^{\prime \prime}\right)$.

Having this reduction in mind, in what follows we may simply assume that the manifold $M$ already satisfies inequality (1). 


\subsection{The configuration space/test map scheme}

Now we reduce Theorem 1.2 to a problem in equivariant topology. Suppose we are given a continuous map

$$
f: \Delta_{N} \longrightarrow M
$$

and a coloring of the vertex set vert $\left(\Delta_{N}\right)=[N+1]=C_{0} \uplus \ldots \uplus C_{m}$ such that the color classes $C_{i}$ are of size $\left|C_{i}\right| \leq r-1$. We want to find a colored Tverberg partition, that is, pairwise disjoint rainbow faces $F_{1}, \ldots, F_{r}$ of $\Delta_{N},\left|F_{j} \cap C_{i}\right| \leq 1$, whose images under $f$ intersect.

The test map $F$ is constructed using $f$ in the following way. Let $f^{* r}:\left(\Delta_{N}\right)^{* r} \longrightarrow \mathbb{Z}_{r} M^{* r}$ be the $r$-fold join of $f$. Since we are interested in pairwise disjoint faces $F_{1}, \ldots, F_{r}$, we restrict the domain of $f^{* r}$ to the simplicial $r$-fold 2-wise deleted join of $\Delta_{N},\left(\Delta_{N}\right)_{\Delta(2)}^{* r}=[r]^{*(N+1)}$. This is the subcomplex of $\left(\Delta_{N}\right)^{* r}$ consisting of all joins $F_{1} * \ldots * F_{r}$ of pairwise disjoint faces. (See [13, Chapter 5.5] for an introduction to these notions.) Since we are interested in colored faces $F_{j}$, we restrict the domain further to the subcomplex

$$
R_{\Delta(2)}^{* r}=\left(C_{0} * \ldots * C_{m}\right)_{\Delta(2)}^{* r}=[r]_{\Delta(2)}^{*\left|C_{0}\right|} * \ldots *[r]_{\Delta(2)}^{*\left|C_{m}\right|} .
$$

This is the subcomplex of $\left(\Delta_{N}\right)^{* r}$ consisting of all joins $F_{1} * \ldots * F_{r}$ of pairwise disjoint rainbow faces. The space $[r]_{\Delta(2)}^{* k}$ is known as the chessboard complex $\Delta_{r, k}[13$, p. 163]. We write

$$
K:=\left(\Delta_{r,\left|C_{0}\right|}\right) * \ldots *\left(\Delta_{r,\left|C_{m}\right|}\right) .
$$

Hence we get a test map

$$
F^{\prime}: K \longrightarrow \mathbb{Z}_{r} M^{* r} .
$$

Let $T_{M^{* r}}:=\left\{\sum_{i=1}^{r} \frac{1}{r} \cdot x: x \in M\right\}$ be the thin diagonal of $M^{* r}$. Its complement $M^{* r} \backslash T_{M^{* r}}$ is called the topological $r$-fold $r$-wise deleted join of $M$ and it is denoted by $M_{\Delta(r)}^{* r}$.

The preimages $\left(F^{\prime}\right)^{-1}\left(T_{M^{* r}}\right)$ of the thin diagonal correspond exactly to the colored Tverberg partitions. Hence the image of $F^{\prime}$ intersects the diagonal if and only if $f$ admits a colored Tverberg partition.

Suppose that $f$ admits no colored Tverberg partition, then the test map $F^{\prime}$ induces a $\mathbb{Z}_{r}$-equivariant map that avoids $T_{M^{* r}}$, that is,

$$
F: K \longrightarrow \mathbb{Z}_{r} M_{\Delta(r)}^{* r} .
$$

We will derive a contradiction to the existence of such an equivariant map using the Fadell-Husseini index theory.

\subsection{The Fadell-Husseini index}

In this section we review equivariant cohomology of $G$-spaces via the Borel construction. This will provide the right tool to prove the non-existence of the test-map (3). We refer the reader to [1, Chap. V] and [10, Chap. III] for more details.

In the following $H^{*}$ denotes singular or Čech cohomology with $\mathbb{F}_{r}$-coefficients, where $r$ is a prime. Let $G$ a finite group and let $E G$ be a contractible free $G$-CW complex, for example the infinite join $G * G * \cdots$, suitably topologized. The quotient $B G:=E G / G$ is called the classifying space of $G$. To every $G$-space $X$ we can associate the Borel construction $E G \times_{G} X:=(E G \times X) / G$, which is the total space of the fibration $X \hookrightarrow E G \times_{G} X \stackrel{p r_{1}}{\longrightarrow} B G$. 
The equivariant cohomology of a $G$-space $X$ is defined as the ordinary cohomology of the Borel construction,

$$
H_{G}^{*}(X):=H^{*}\left(E G \times_{G} X\right) .
$$

If $X$ is a $G$-space, we define the cohomological index of $X$, also called the Fadell-Husseini index [11], [12], to be the kernel of the map in cohomology induced by the projection from $X$ to a point,

$$
\operatorname{Ind}_{G}(X):=\operatorname{ker}\left(H_{G}^{*}(\mathrm{pt}) \stackrel{p^{*}}{\longrightarrow} H_{G}^{*}(X)\right) \subseteq H_{G}^{*}(\mathrm{pt}) .
$$

The cohomological index is monotone in the sense that if there is a $G$-map $X \longrightarrow_{G} Y$ then

$$
\operatorname{Ind}_{G}(X) \supseteq \operatorname{Ind}_{G}(Y) .
$$

If $r$ is odd then the cohomology of $\mathbb{Z}_{r}$ with $\mathbb{F}_{r}$-coefficients as an $\mathbb{F}_{r}$-algebra is

$$
H^{*}\left(\mathbb{Z}_{r}\right)=H^{*}\left(B \mathbb{Z}_{r}\right) \cong \mathbb{F}_{r}[x, y] /\left(y^{2}\right),
$$

where $\operatorname{deg}(x)=2$ and $\operatorname{deg}(y)=1$. If $r=2$ then $H^{*}\left(\mathbb{Z}_{r}\right) \cong \mathbb{F}_{2}[t], \operatorname{deg} t=1$.

The index of the configuration space $K$, defined in (2), was computed in [8, Corollary 2.6]:

Theorem 2.2 $\operatorname{Ind}_{\mathbb{Z}_{r}}(K)=H^{*} \geq N+1\left(B \mathbb{Z}_{r}\right)$.

Therefore in the proof of Theorem 1.2 it remains to show that $\operatorname{Ind}_{\mathbb{Z}_{r}}\left(M_{\Delta(r)}^{* r}\right)$ contains a non-zero element in dimension less or equal to $N$. Indeed, the monotonicity of the index (4) then implies the non-existence of a test map (3), which in turn implies the existence of a colored Tverberg partition.

Let us remark that the index of $K$ becomes larger with respect to inclusion than in Theorem 2.2 if just one color class $C_{i}$ has more than $r-1$ elements. That is, in this case our proof of Theorem 1.2 does not work anymore. In fact, for any $r$ and $d$ there exist $N+1$ colored points in $\mathbb{R}^{d}$ such that one color class is of size $r$ and all other color classes are singletons that admit no colored Tverberg partition.

\subsection{The index of the deleted join of the manifold}

In this section we prove that $\operatorname{Ind}_{\mathbb{Z}_{r}} M_{\Delta(r)}^{* r}$ contains a non-zero element in degree $N$. Together with Theorem 2.2 we deduce that $\operatorname{Ind}_{\mathbb{Z}_{r}} M_{\Delta(r)}^{* r}$ is not contained in $\operatorname{Ind}_{\mathbb{Z}_{r}}(K)$, hence by the monotonicity of the index, the test-map (3) does not exist, which finishes the proof.

We have inclusions

$$
T_{M^{* r}} \hookrightarrow\left\{\sum \lambda_{i} x \in M^{* r}: \lambda_{i}>0, \sum \lambda_{i}=1, x \in M\right\} \cong M \times \Delta_{r-1}^{\circ} \hookrightarrow M^{* r},
$$

where $\Delta_{r-1}^{\circ}$ denotes the open $(r-1)$-simplex. Since $M$ is a smooth $\mathbb{Z}_{r}$-invariant manifold, $T_{M^{* r}}$ has a $\mathbb{Z}_{r}$-equivariant tubular neighborhood in $M^{* r}$; see [6, Section VI.2]. Its closure can be described as the disk bundle $D(\xi)$ of an equivariant vector bundle $\xi$ over $M$. We denote its sphere bundle by $S(\xi)$. The fiber $F$ of $\xi$ is as a $\mathbb{Z}_{r}$-representation the $(d+1)$-fold sum of $W_{r}$, where $W_{r}=\left\{x \in \mathbb{R}\left[\mathbb{Z}_{r}\right]: x_{1}+\ldots+x_{r}=0\right\}$ is the augmentation ideal of $\mathbb{R}\left[\mathbb{Z}_{r}\right]$.

The representation sphere $S(F)$ is of dimension $N-1$. It is a free $\mathbb{Z}_{r}$-space, hence its index is

$$
\operatorname{Ind}_{\mathbb{Z}_{r}}(S(F))=H^{* \geq N}\left(B \mathbb{Z}_{r}\right) .
$$


This can be directly deduced from the Leray-Serre spectral sequence associated to the Borel construction $E \mathbb{Z}_{r} \times_{\mathbb{Z}_{r}} S(F) \rightarrow B \mathbb{Z}_{r}$, noting that the images of the differentials to the bottom row give precisely the index of $S(F)$. The latter can be seen from the edge-homomorphism. For background on Leray-Serre spectral sequences we refer to [14, Chapters 5,6].

The Leray-Serre spectral sequence associated to the fibration $S(\xi) \rightarrow M$ collapses at $E_{2}$, since $N=$ $(r-1)(d+1) \geq d+1$ and hence there is no differential between non-zero entries. Thus the map $i^{*}: H^{N-1}(S(\xi)) \rightarrow H^{N-1}(S(F))$ induced by inclusion is surjective.

The Mayer-Vietoris sequence associated to the triple $\left(D(\xi), M_{\Delta(r)}^{* r}, M^{* r}\right)$ contains the subsequence

$$
H^{N-1}\left(M_{\Delta(r)}^{* r}\right) \oplus H^{N-1}(D(\xi)) \stackrel{j^{*}+k^{*}}{\longrightarrow} H^{N-1}(S(\xi)) \stackrel{\delta}{\longrightarrow} H^{N}\left(M^{* r}\right) .
$$

We see that $H^{N}\left(M^{* r}\right)$ is zero: This follows from the formula

$$
\widetilde{H}^{*+(r-1)}\left(M^{* r}\right) \cong \widetilde{H}^{*}(M)^{\otimes r}
$$

as long as $N-(r-1)>r e$, where $e$ is the cohomological dimension of $M$. This inequality is equivalent to $d>\frac{r}{r-1} e$, which can be assumed by applying the reduction from Section 2.1 at least $\left\lfloor 1+\frac{e}{r-1}\right\rfloor$ times. Hence we can assume that $H^{N}\left(M^{* r}\right)=0$.

Furthermore inequality 11 implies that $N-1 \geq d>\operatorname{cohdim}(M)$. Hence the term $H^{N-1}(D(\xi))=$ $H^{N-1}(M)$ of the sequence is zero as well.

Thus the map $j^{*}: H^{N-1}\left(M_{\Delta(r)}^{* r}\right) \rightarrow H^{N-1}(S(\xi))$ is surjective. Therefore the composition $(j \circ$ $i)^{*}: H^{N-1}\left(M_{\Delta(r)}^{* r}\right) \rightarrow H^{N-1}(S(F))$ is surjective as well. We apply the Borel construction functor $E \mathbb{Z}_{r} \times_{\mathbb{Z}_{r}}(-) \rightarrow B \mathbb{Z}_{r}$ to this map and apply Leray-Serre spectral sequences; see Figure 3 .

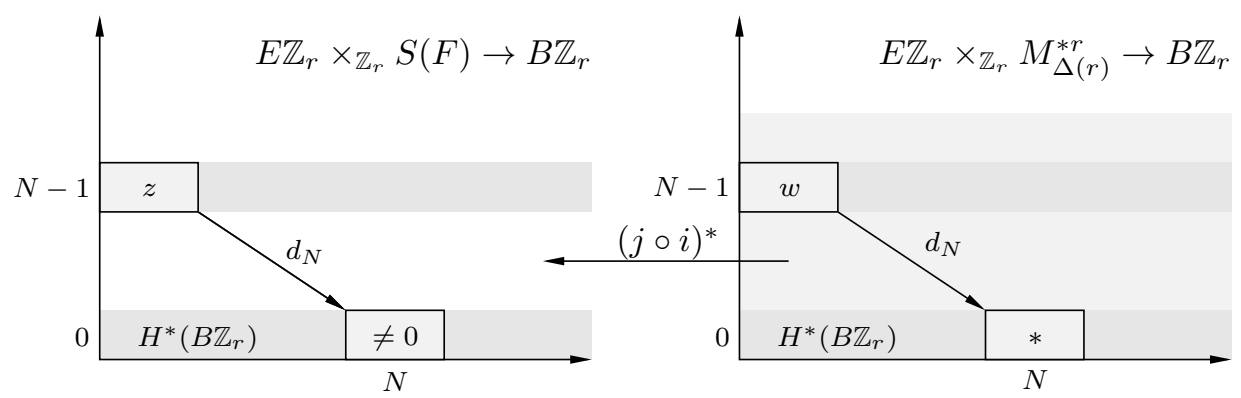

Fig. 3: We associate to the map $S(F) \stackrel{j \circ i}{\longrightarrow} M_{\Delta(r)}^{* r}$ the Borel constructions and spectral sequences to deduce that $M_{\Delta(r)}^{* r}$ contains a non-zero element in dimension $N$.

At the $E_{2}$-pages, the generator $z$ of $H^{N-1}(S(F))$ has a preimage $w$ since $(j \circ i)^{*}$ is surjective. At the $E_{N}$-pages $(j \circ i)^{*}\left(d_{N}(w)\right)=d_{N}(z)$, which is non-zero by $(5)$. Hence $d_{N}(w) \neq 0$, which is an element in the kernel of the edge-homomorphism $H^{*}\left(B \mathbb{Z}_{r}\right) \rightarrow H_{\mathbb{Z}_{r}}^{*}\left(M_{\Delta(r)}^{*}\right)$.

Therefore, the index of $M_{\Delta(r)}^{* r}$ contains a non-zero element in dimension $N$. This completes the proof of Theorem 1.2 if $f$ can be extended to $\Delta^{N}$. 


\section{References}

[1] A. Adem, J. Milgram. Cohomology of Finite Groups, Grundlehren der mathematischen Wissenschaften, Vol. 309, Springer 1994.

[2] I. Bárány, Z. Füredi, L. Lovász. On the number of halving planes, Combinatorica 10, 1990, 175-183.

[3] I. Bárány, D. G. Larman. A colored version of Tverberg’s theorem, J. London Math. Soc. 45, 1992, 314-320.

[4] I. Bárány, S. B. Shlosman, A. Szúcs. On a topological generalization of a theorem of Tverberg, J. London Math. Soc. 23, 1981, 158-164.

[5] B. J. Birch. On 3N points in the plane, Math. Proc. Cambridge Phil. Soc. 55, 1959, 289-293.

[6] G. E. Bredon. Introduction to Compact Transformation Groups, New York, Academic Press, 1972.

[7] P. V. M. Blagojević, B. Matschke, G. M. Ziegler. Optimal bounds for the colored Tverberg problem, arxiv:0910.4987v2, 2009, 11 pages.

[8] P. V. M. Blagojević, B. Matschke, G. M. Ziegler. Optimal bounds for a colorful Tverberg-Vrećica type problem, Adv. Math. 226 (6), 2011, 5198-5215.

[9] P. V. M. Blagojević, B. Matschke, G. M. Ziegler. A tight colored Tverberg theorem for maps to manifolds, preprint, 2010, 7 pages.

[10] T. tom Dieck. Transformation Groups, de Gruyter Studies in Math., Vol. 8, Berlin, 1987.

[11] E. Fadell, S. Husseini. Relative cohomological index theories, Adv. Math. 64, 1987, 1-31.

[12] E. Fadell, S. Husseini. An ideal-valued cohomological index theory with applications to BorsukUlam and Bourgin-Yang theorems, Ergodic Theory and Dynamical Systems 8*, 1988, 73-85.

[13] J. Matoušek. Using the Borsuk-Ulam Theorem. Lectures on Topological Methods in Combinatorics and Geometry, Universitext, Springer 2003.

[14] J. McCleary. A User's Guide to Spectral Sequences, Cambridge University Press, second edition, 2001.

[15] H. Tverberg. A generalization of Radon's theorem, J. London Math. Soc. 41, 1966, 123-128.

[16] A. Yu. Volovikov. On a topological generalization of the Tverberg theorem, Math. Notes 59, No. 3, 1996, 324-326.

[17] S. Vrećica, R. Živaljević. The colored Tverberg's problem and complex of injective functions, J. Combin. Theory Ser. A, 61, 1992, 309-318. 